\title{
1967. Une thèse en Sorbonne : L'Analyse mathématique des faits sociaux
}

1967. A PhD thesis defense at the Sorbonne: L'Analyse mathématique des faits sociaux

Jean-Paul Grémy

\section{OpenEdition}

\section{Journals}

Édition électronique

URL : https://journals.openedition.org/ress/4194

DOI : $10.4000 /$ ress.4194

ISSN : 1663-4446

Éditeur

Librairie Droz

Édition imprimée

Date de publication : 14 décembre 2018

Pagination : 13-40

ISSN : 0048-8046

Référence électronique

Jean-Paul Grémy, «1967. Une thèse en Sorbonne : L'Analyse mathématique des faits sociaux », Revue européenne des sciences sociales [En ligne], 56-2 | 2018, mis en ligne le 14 décembre 2021, consulté le 06 janvier 2022. URL : http://journals.openedition.org/ress/4194 ; DOI : https://doi.org/10.4000/ress 4194

(C) Librairie Droz 


\section{UNE THĖSE EN SORBONNE: L'ANALYSE MATHÉMATIQUE DES FAITS SOCIAUX}

JEAN-PAUL GRÉMY

Paris, ENS - EHESS - CNRS, Centre Maurice Halbwachs jean-paul.gremy@orange.fr

Résumé. En 1967, date de la publication de L'Analyse mathématique des faits sociaux, l'utilisation des mathématiques dans les sciences sociales faisait encore l'objet de fortes réticences. Ce texte décrit les causes et les formes de cette opposition, et retrace la manière dont le livre de Raymond Boudon fut reçu par les sociologues et contribua au développement d'une sociologie scientifique.

Mots-clés: analyse causale, analyse de dépendance, mathématiques en sociologie.

Abstract. In 1967, when Raymond Boudon published L'Analyse mathématique des faits sociaux, social scientists were still very reluctant to resort to mathematics. In this paper, I describe the causes and the forms of this, I recall how this book was received by sociologists, and the important role it played in the development of sociology as a science.

Keywords: causal analysis, mathematical sociology, path analysis. 
En 1967, lorsque paraît L'Analyse mathématique des faits sociaux, thèse soutenue le I8 mars de la même année dans la salle Louis Liard de la Sorbonne', Raymond Boudon a déjà publié plus d'une quinzaine d'articles scientifiques, une contribution à un ouvrage, et deux anthologies commentées (avec Paul F. Lazarsfeld). Dans leur quasi-totalité, ces publications ont en commun un contenu méthodologique et technique affirmé. À cette date, l’importance de L'analyse mathématique des faits sociaux tient à trois caractéristiques: c'est le premier livre entièrement rédigé par Boudon; il constitue sa thèse principale de Doctorat d'État (étape déterminante dans une carrière universitaire) ; et il comble une lacune dans la littérature sociologique française de l'après Seconde Guerre mondiale. Pourtant, dans la plupart des biographies de Boudon, et dans les in memoriam qui ont suivi son décès, ou bien cet ouvrage n’est pas mentionné, ou bien il occupe une place mineure², éclipsé qu’il est par L’inégalité des chances et les nombreuses publications qui ont suivi.

Ce relatif effacement est corroboré, trente-cinq ans après, par Olivier Martin. Citant explicitement les premiers travaux de Boudon, il écrit : «En France, la période d’après-guerre est également marquée par la volonté de quelques chercheurs de donner une base mathématique à l'analyse de quelques faits sociaux [...]. Ces efforts restent toutefois relativement isolés ». Dans l'après-guerre, les sociologues qui analysent des données quantitatives utilisent en effet de préférence les outils statistiques et probabilistes hérités des psychologues, utilisation qui commence alors à être facilitée par les progrès de l'informatique (Martin, 2002, p. 6).

I À cette époque, la Sorbonne, dont le bâtiment abrite alors la Faculté des Lettres et la Faculté des Sciences de l'Université de Paris, jouit d'une grande renommée à la fois sur le plan national et à l'étranger. Après mai 1968, son démantèlement en unités diverses rattachées à de multiples universités et l'accroissement considérable du nombre d'étudiants et d'enseignants (Prost, 1989, p.62-63) diminueront considérablement son prestige.

2 Sur une dizaine d'In memoriam consultés, seuls quatre mentionnent L'Analyse mathématique: ceux de Salvador Giner (2013), de François Héran (2013), de Sylvie Mesure (2013) et de Louis-André Vallet (2013). 
Pour comprendre ce désintérêt apparent, il est nécessaire de rappeler quel était, au milieu des années 1960, l'état de la discipline : les conditions de recrutement et de formation des sociologues, le contenu de cette formation, les avancées de la recherche en sciences sociales. Il sera alors possible d'évaluer l'apport méthodologique de l'ouvrage lors de sa parution, et de comprendre la manière dont il a été reçu par la communauté des sociologues.

\section{LA SITUATION DE LA SOCIOLOGIE FRANÇAISE EN 1967}

«En 1945, la sociologie française est au plus bas: aucune revue marquante, une poignée de postes universitaires placés dans l'orbite de la philosophie, pratiquement pas de recherche collective organisée» (Chenu, 2002, p.46). Vingt ans après, la situation a sensiblement évolué : en 1966-1967, on dénombre une soixantaine d'enseignants de sociologie titulaires et près de 250 chercheurs (en comptant les centres à financement privé), dont une centaine de chercheurs CNRS (Drouard, 1982, p. 76 ; Chenu, 2002, p. 47, graphique I, et p. 49). En outre, la licence de sociologie et le doctorat de troisième cycle ont été créés en 1958.

\section{I.I. LE CENTRE D'ÉTUDES SOCIOLOGIQUES \\ ET LES DÉBUTS DE LA RECHERCHE EN SOCIOLOGIE}

En 1967, malgré l'existence d'un enseignement de sociologie dans les Facultés de lettres et sciences humaines et à l'École pratique des hautes études (VI ${ }^{\mathrm{e}}$ section), bon nombre de chercheurs et d'enseignants-chercheurs en sociologie ne sont pas de «purs sociologues». Leur formation de base a été acquise dans une autre discipline: philosophie, psychologie, plus rarement histoire, économie, démographie, droit, sciences politiques, voire, plus rarement encore, statistiques, mathématiques et sciences de l'ingénieur; ils ont donc tous fait, en quelque sorte, leur apprentissage de sociologue «sur le tas» (Chenu, 2002, p. 46).

Le Centre d'études sociologiques (CES) a été créé en 1946; mais à cette époque, «l'activité principale du nouveau centre n'était pas la recherche, mais l'organisation de conférences [...]. Elles avaient pour but, essentiellement, de stimuler l'intérêt vis-à-vis des sciences sociales et de combattre l'indifférence et même l'hostilité à leur égard [...]. Dans les grands courants intellectuels de l'époque, les préoccupations 
sociologiques étaient plus ou moins suspectes» (Heilbron, 1991, p. 367). Les conférences n'ayant pas obtenu un grand succès, et le CNRS étant en mesure d'engager plus de chercheurs que par le passé, les activités de recherche commencèrent à se développer (ibid., p. 369). Mais ces recherches souffraient, du moins au début, de nombreux handicaps: faiblesse des motivations et absence de statut pour les chercheurs, confusion entre recherche et militantisme, manque de formation et manque de moyens, absence de ligne directrice et de paradigme unificateur (ibid., p. 370-373; Tréanton, 199I, p. 390-392; Marcel, 2005, p. 4-5). En outre, il existait alors au CES un respect de la «division du travail», selon laquelle les «grandes» questions, théoriques et autres, étaient réservées aux professeurs (Heilbron, 1991, p. 37I).

En une dizaine d'années, la recherche est toutefois parvenue à se développer (Laude, I960, p. 94). Mais les chercheurs du CES avaient conscience de leurs insuffisances sur les plans technique et méthodologique. À partir de 1954, ils ont commencé à mettre en place des formations internes aux techniques de terrain : entretien et analyse de contenu, statistiques, méthodes d'échantillonnage, etc.

[Dans ces formations] ce sont les chercheurs les plus expérimentés qui initient dans un premier temps les novices aux techniques d'enquêtes [...]. En échangeant entre eux conseils méthodologiques et compétences en matière de sociologie anglo-saxonne, [les chercheurs du CES] ont posé cahin-caha des jalons dans leurs champs de recherche respectifs qui se sont peu à peu autonomisés à mesure que l'on rôdait techniques et résultats [...]. Il faudra néanmoins attendre les années 1960 et presque une génération, pour qu'avec des auteurs comme Michel Crozier, Alain Touraine, Pierre Bourdieu ou Raymond Boudon naissent de nouveaux paradigmes, comme s'il avait fallu une décennie pour que tous ces nouveaux apprentissages soient «digérés » et systématisés (Marcel, 2005, p. 8-10).

\section{I.2. L'ENSEIGNEMENT DE LA SOCIOLOGIE}

La faculté des Lettres de Paris (Sorbonne) avait créé dès la Libération un certificat libre de sociologie; mais ce dernier n'avait pas pour objectif de former des sociologues (Chenu, 2002, p. 48). Créée en 1958 à l'initiative de Raymond Aron, la licence de sociologie «marque l'ambition pour la discipline de déboucher sur l'exercice d'une activité professionnelle en dehors du monde universitaire et des organismes de recherche» (ibid.). Le décret du 2 avril 1958 
précise que la licence de sociologie comporte quatre certificats: sociologie générale, psychologie sociale, économie politique et sociale et un certificat dit optionnel, à choisir dans une liste assez étendue : ethnologie, démographie, géographie humaine, etc. (Drouard, 1982, p. 74, note 55).

Comme le rappelle Boudon (Assogba, 1999, p. 132), à l'époque, l'enseignement de la sociologie à la Sorbonne était dominé par deux pôles: Georges Gurvitch et Claude Lévi-Strauss. C'est pourquoi il a choisi de partir à la découverte de la sociologie nord-américaine :

J’avais décidé de faire un stage chez Lazarsfeld à l'issue de mon service militaire, parce que j'avais découvert un peu par hasard sur les rayons de la bibliothèque de la rue d'Ulm The Language of Social Research. À tort ou à raison, ce livre m'était apparu comme offrant une alternative à la sociologie gurvitchienne alors régnante en France, que j'avais toujours soupçonnée de dissimuler beaucoup de banalités dans des maquis de typologies et de définitions tatillonnes, et à sa concurrente structuraliste, qui me paraissait sujette à caution, tant par son ambition totalisante que par son platonisme (Boudon, 2001a, p. 19).

Après sa nomination à la Sorbonne en 1955 comme professeur de psychologie sociale, Jean Stoetzel avait contribué à briser ce monopole : «La sociologie gurvitchienne, bien quelle se définisse comme un "hyper-empirisme", s'inscrit dans cette tradition académique. Sa vigoureuse emprise sur la vieille Sorbonne est battue en brèche par quelques sociologues plus tournés vers l'enquête, au premier rang desquels Jean Stoetzel, qui introduisit à l'IFOP et à l'INED les méthodes de sondage mises au point aux États-Unis dans les années 1930 » (Chenu, 2002, p. 48). Cet élargissement sera amplifié par la nomination d’Aron, comme chargé d'enseignement de sociologie (en 1955) puis comme professeur (en 1958).

Mais le contenu effectif des enseignements de la licence de sociologie ne dépendait pas seulement des programmes officiels et des orientations personnelles des professeurs; il était aussi tributaire des moyens relativement limités alloués à cette discipline, en encadrement comme en matériel. Cela était particulièrement sensible pour la formation méthodologique et technique (activités de terrain, et traitement informatique des données). 


\section{I.3. LA PLACE DES MATHÉMATIQUES ET DE L'INFORMATIQUE} DANS L'ENSEIGNEMENT DE LA SOCIOLOGIE

Il existait encore, au début des années 1960, chez certains enseignants en sociologie, un fort préjugé contre l'utilisation des méthodes quantitatives dans les sciences sociales. S’abritant derrière l'évidence que «jamais l'on ne pourra réduire l'homme en équations », ces contempteurs de l'«arithmomanie » allaient parfois jusqu'à proclamer : «sociologie quantitative = sociologie capitaliste $\gg^{4}$. Gurvitch lui-même, dans son Traité de sociologie, avait repris à son compte l'anathème de Pitirim Sorokin contre la «quantophrénie». Dans la première édition de La vocation actuelle de la sociologie, il affirmait: «La Masse en tant que forme de sociabilité [...] est absolument indépendante de toute résultante d'un quelconque calcul statistique [...]. L’exemple déplorable des soidisant "sondages" de l'opinion publique à la manière de Gallup semble spécialement fait pour prouver toute la vanité des calculs statistiques de moyennes non adaptées à des cadres sociaux concrets » (Gurvitch, 1950, p. I30). Dans la seconde édition, s’il tempérait son propos initial et n’attaquait plus nommément Gallup (afin soit d'ignorer délibérément, soit de ménager son collègue Stoetzel, nouvellement élu à la Sorbonne), il n’en déclarait pas moins :

Les statistiques, faute d'être appliquées à un cadre social réel soigneusement vérifié et délimité, ne représentent que des manipulations purement mathématiques avec les grands nombres [...]. Les sondages ne peuvent s'accomplir dans le vide et présupposent, pour ne pas devenir dérisoires, des unités collectives réelles, partielles et globales, dont on connaisse préalablement l'engrenage, y

3 Dans un texte écrit en décembre 1968, évoquant les progrès de la comptabilité sociale, le développement des enquêtes sociales, la multiplication des sondages d'opinion et la création des banques de données, Boudon écrit: «Certes, des esprits chagrins s'inquiètent de ces développements. Les uns proclament les méfaits politiques de sondages, les autres craignent que la standardisation de l'information déshumanise la recherche, dénoncent les abus des "questionnaires" et autres formes de techniques quantitatives en sociologie et proposent de revenir à des méthodes d'observation plus proches du "vécu". Si ces dénonciations ont peut-être dans certains cas un fondement, elles sont surtout un symptôme de la réaction obscurantiste dont l'histoire nous montre qu'elle accompagne généralement toute mutation scientifique» (Boudon, 197lb, p. 47).

4 Reproduisant ainsi l'erreur de Joseph Staline qui, en qualifiant l'informatique de «science bourgeoise», a fortement freiné le développement de cette discipline en URSS, en dépit des premières recherches prometteuses des pionniers russes du calcul automatique. 
compris l'accentuation des couches en profondeur. Les questionnaires et les entrevues ne donnent un résultat que si l'on part d'hypothèses valables et ils réussissent plutôt à démolir de fausses présuppositions qu'à conduire à des découvertes (Gurvitch, 1957, p. 13).

Il est vrai que, dans son Traité de sociologie, il inclut un court chapitre sur «Les problèmes de la statistique», rédigé par Georges-Théodule Guilbaud (Gurvitch, 1958, p. I14-I34); mais il s'agit essentiellement d'un exposé historique, non technique.

En dépit de l'influence de ce courant, de nombreuses initiatives avaient contribué à développer la formation des étudiants en sciences humaines, d’abord aux statistiques appliquées, puis aux mathématiques élémentaires. Les psychosociologues avaient joué un rôle important dans la formation des chercheurs du CES (Marcel, 2005, p. 8-9). Comme la psychologie s'était, bien avant la sociologie, dégagée de la tutelle de la philosophie pour se constituer comme science, ce sont les étudiants dans cette discipline qui ont bénéficié les premiers d'un enseignement de statistiques, en particulier grâce à Jean-Marie Faverge (1950). Un premier fascicule d'initiation, par Roger Daval et Georges-Théodule Guilbaud avait aussi été publié en 1950, mais il n’a malheureusement pas eu de suite. Par ailleurs, à partir de 1955-1956, Maurice Reuchlin a professé, à l'Institut de psychologie de l'université de Paris, un cours de «statistique notionnelle» ne faisant appel à aucune formalisation, et par conséquent plus accessible aux étudiants (cours publié dans le Bulletin de psychologie). Dans ce cours, il explicitait les postulats, et donc les conditions d'emploi, des diverses techniques usuelles, préfigurant sa thèse de doctorat (Reuchlin, 1962). Enfin, le Précis de statistique de Stéphane Ehrlich et Claude Flament (196I) constituait une bonne initiation, très pédagogique, à l'intention des psychologues. Mais les statistiques appliquées à la psychologie se référaient généralement à des situations expérimentales, dans lesquelles il est possible de manipuler certaines variables (selon un plan d'expérience) et d'analyser finement leurs effets directs et leurs interactions (analyse de la variance et tests de significativités); leur

5 D'où, chez les sociologues, la religion du test du $\chi^{2}$, considéré alors comme un gage de «scientificité». 
application aux données d'observation recueillies par les sociologues pouvait donc être source d'erreurs et de difficultés particulières.

En mai 1962, à l'initiative de Guilbaud et de Marc Barbut (du Groupe de mathématiques sociales et de statistique de l'EPHE ${ }^{6}$ ), un colloque sur l'enseignement des mathématiques et de la statistique pour les sciences humaines s'est tenu à Paris afin de coordonner les divers enseignements utilisant ces techniques. Dans les années qui suivirent, ont paru quelques ouvrages spécialisés (Flament, 1965; Matalon, 1965), et les enseignements se sont développés. Mais ceux-ci se sont heurtés à des problèmes pédagogiques, liés à la disparité des deux cultures, mathématique d'un côté et sciences humaines de l'autre. Guilbaud préconisait alors la méthode de «l'évangélisation par le clergé indigène »: s'il est vrai que le prêtre autochtone n’est pas nécessairement expert en théologie, il présente l'avantage essentiel de parler la langue des catéchumènes. De même, une initiation faite par des spécialistes des sciences humaines, ayant quelques notions élémentaires de mathématiques et de statistiques, pourra être plus facilement assimilée par les étudiants ; mais comme elle n’est pas à l’abri d'erreurs ou d'approximations, cette initiation devra être consolidée et approfondie par des mathématiciens. Cette méthode n’a malheureusement pas pu être généralisée, faute d'enseignants qualifiés. Parmi les efforts pour initier les futurs sociologues aux mathématiques, il faut également mentionner les films pédagogiques de Georges-Théodule et Pierre Guilbaud, qui n’ont pas connu la diffusion qu’ils auraient méritée. Enfin, en 1967, paraît le premier tome du manuel de Barbut.

Pour les étudiants avancés et les chercheurs, il existait heureusement d'autres sources d'initiation relativement accessibles. La Méthode statistique d'Eugène Morice et Fernand Chartier (1954) est un manuel très clair, plutôt destiné aux économistes, mais constituant une bonne présentation des probabilités et des statistiques; il ne présuppose que des connaissances mathématiques du niveau baccalauréat. On trouvait également des éléments de mathématique ou de statistique appliquées dans divers manuels de méthodologie traduits de l’anglais, et en parti- 
culier dans celui de John G. Kemeny, J. Laurie Snell et Gerald L. Thomson (1960). Enfin, pour ceux qui lisaient l'anglais, parmi de nombreux titres, le livre de base était naturellement l'Introduction to Mathematical Sociology, de James S. Coleman (1964).

Par ailleurs, il faut se rappeler qu’à la fin des années 1960, les microordinateurs n'existaient pas encore et que l'informatique était à l'âge des cartes perforées et des gros systèmes relativement lents. Il n'y avait alors que quelques sociologues sachant programmer (parmi lesquels Boudon) et les logiciels conçus spécialement pour les traitements statistiques dans les sciences humaines (comme Osiris, PVS ou Daphné) étaient encore peu répandus.

\section{LE CONTENU DE}

\section{L'ANALYSE MATHÉMATIQUE DES FAITS SOCIAUX}

Dans les premières phases du développement des sciences sociales en France après la Libération, deux références ont joué un rôle essentiel : la référence aux sciences exactes et naturelles, qui incarnent un «modèle de scientificité», et la référence aux États-Unis, pour l'apprentissage des techniques et méthodes d'enquêtes (Drouard, 1982, p.60, 62). La publication du livre de Boudon en I967 peut être considérée comme l'incarnation de ces deux tendances.

\section{I. LA GENÈSE DU LIVRE}

Boudon rapporte en ces termes la genèse de L’Analyse mathématique:

Le point de départ de ce livre a été une réflexion sur le texte de Lazarsfeld: «Interpretation of statistical relations as a research operation». Il s'agit d'un texte célèbre, d'une sorte de code de la recherche dite «empirique» en sociologie [...]. Si on le considère de manière très générale, il fait apparaittre que l'interprétation sociologique des tableaux de contingence qu'on établit à partir des données d'une recherche consiste naturellement à leur associer une structure causale [...]. Les tables de contingence présentées par Lazarsfeld dans ses trois exemples concernent trois variables dichotomiques [...]. La question qui vient naturellement à l'esprit est la suivante: supposons que quatre variables ou davantage soient impliquées dans un même réseau de relations. Ne peut-on améliorer ou

7 Voir par exemple la description que Boudon fait du dépouillement d'une enquête sur ordinateur au début des années 1970 (Boudon, 197Ic). 
modifier l'interprétation tirée des propriétés des distributions à trois variables en considérant les propriétés de la distribution de ces quatre variables ou plus, prises simultanément? Cette question ne reçoit pas de réponse au niveau de la méthodologie de type intuitif préconisée par Lazarsfeld [...]. Le principe une fois admis que l'interprétation des résultats d'une enquête consiste le plus souvent à analyser les propriétés des distributions de manière à déceler la présence d'une structure causale, comment étendre la méthodologie de Lazarsfeld de manière à traiter du cas général où non pas 3 , mais $n$ variables apparaissent comme interconnectées? Très vite, je me suis rendu compte que les situations impliquant un petit nombre de variables (3 ou 4) jouent un peu le rôle du pentagone ou de l'hexagone de Descartes en ce qu'elles marquent les limites assignées à l'«imagination» : jusqu'à trois ou quatre variables, l'intuition suffit à conduire une analyse correcte [...]. Au-delà, la lecture directe devient difficile, puis pratiquement impossible. Il faut alors inverser la procédure: c'est-à-dire faire l'hypothèse que les données recueillies peuvent être expliquées par une structure causale particulière et vérifier que cette structure : I) est effectivement compatible avec les données, 2) en rend mieux compte que tout autre [...]. Bref, quand on passe du cas de 3 ou 4 variables à des cas plus complexes, il faut troquer la méthode directe ou déductive contre une méthode indirecte ou inductive utilisant une instrumentation de plus en plus familière au sociologue : celle des modèles. En effet, la suite des opérations impliquées par la lecture indirecte suppose une traduction mathématique des hypothèses causales (Boudon, 1967b, p. 389-39I).

\subsection{LES GRANDES LIGNES DE L'OUVRAGE}

L’objet du livre est annoncé clairement dès l'introduction :

Le présent ouvrage traite des apports de la pensée formelle à l'analyse des enquêtes, des sondages et des relevés statistiques de la comptabilité sociale qui se proposent à l'attention du sociologue. Il concerne donc [...] la seule sociologie d'observation [...]. Formulé de la manière la plus abstraite, le problème dont nous traiterons ici est celui des relations entre les attributs ou, plus généralement, les variables caractérisant les membres d'une population, lorsque ces attributs ou variables ont été déterminés à partir d'une situation d'observation (Boudon, 1967a, p. II). 
Il se propose par conséquent d’aborder l'essentiel des problèmes méthodologiques généraux qui se posent aux sociologues, à l'exclusion de l'analyse des données expérimentales (qui propose des modèles de structures trop simples, peu adaptées à la complexité des données d'observation) et des modèles théoriques spéculatifs (situations délibérément idéalisées, comme la théorie des jeux), mais aussi de l'analyse formelle des structures sociales (déjà traitée par Claude Flament). Son contenu est centré sur trois types d'opérations : I) expliquer les relations entre variables en termes de causalité; 2) subsumer une multitude de caractères particuliers sous un petit nombre de catégories ; 3) analyser les processus sociaux.

Les deux premières parties, qui représentent près de la première moitié du texte (46\%), sont consacrées explicitement à l'analyse causale, c'est-à-dire à l'élaboration de modèles explicatifs visant à rendre compte d'un ensemble de données d'observation. Ce type de modèles distingue deux grandes catégories de variables (ou d’attributs): variables dépendantes (à expliquer), et variables indépendantes, dont certaines sont supposées avoir une influence sur les premières (variables explicatives). Le point de départ en est le schéma durkheimien d'inférence causale à partir de corrélations statistiques à l'œuvre dans Le Suicide. L'auteur présente ensuite des structures causales plus complexes, introduit la notion d'effets d'interaction et met en parallèle les deux principales techniques applicables, l'analyse de régression et l'analyse de dépendance $^{8}$. Il aborde enfin le problème de l'analyse écologique (c'est-à-dire de l'inférence à des propositions sur des individus, de corrélations observées entre des variables globales) et en souligne les difficultés d'interprétation.

8 Dans la réédition corrigée de L'Analyse mathématique, il précise: «La méthode que nous présentons ici sous le nom d'analyse de dépendance est en fait une synthèse ou une intégration logique de la path analysis de Wright et des travaux des sociologues que nous avons examinés au chapitre précédent» (Boudon, 1970, p.97). L'analyse de dépendance se distingue de l'analyse de régression par l'introduction de l'hypothèse de non-corrélation entre les facteurs implicites. «Si nous avons jugé utile de lui donner un nom différent, c'est, d'une part, que cette hypothèse permet d'introduire des algorithmes spécifiques et, d'autre part, qu'elle nous paraît être, moins un cas particulier de l'analyse de régression qu'une méthode générale de traitement des structures causales complexes» (Boudon, 1967a, p. 133). 
La troisième partie porte sur la subsomption de caractères particuliers sous un petit nombre de concepts généraux; elle est peu développée (12\% du texte). Elle traite surtout des différents types d'analyse factorielle, ainsi que de l'analyse hiérarchique (construction d'échelles) et présente très succinctement les méthodes non métriques d’analyse dimensionnelle. Bien qu'il utilise le terme, l'auteur n’aborde pas l'ensemble des méthodes de construction de typologies proprement dites, ni certaines techniques (comme la segmentation) dont un sous-produit peut être la définition de types.

Enfin, l’analyse des données recueillies dans le temps (séries chronologiques, données de panels) représente 39\% du texte (quatrième partie). Les spécificités et les limites de nombreux modèles d'analyse des processus sociaux sont décrites en détail (modèles de diffusion, de contagion; tables de rotation; processus de Markov). Les derniers développements portent sur les modèles simulés, particulièrement intéressants lorsque la théorie explicative est trop complexe pour être présentée sous la forme d'un ensemble d'équations mathématiques solubles, ou lorsque l'on désire introduire dans le modèle une part de hasard (modèles stochastiques).

\subsection{LES APPORTS TECHNIQUES}

Sur le plan strictement technique, l'un des intérêts de ce livre est de constituer en quelque sorte un catalogue raisonné de la plupart des techniques d’analyse de données quantitatives ayant fait leurs preuves, nourri d'exemples d’application tirés de la littérature scientifique, et signalant les limites et les conditions d'emploi de ces techniques. Dans certains cas, l'auteur propose même des améliorations ou des rectifications. Il mentionne aussi des techniques relativement récentes, comme le déploiement (unfolding technique) de Clyde C. Coombs, ou l'analyse de l'incertitude de William J. McGill.

D’autre part, bien que l'informatique n'en soit encore qu'à ses débuts, l'auteur exprime à plusieurs reprises son espoir que l'ordinateur puisse bientôt jouer un rôle important dans la recherche sociologique. Il souligne l'intérêt de traduire «en un langage accessible à un ordinateur» les techniques d'analyse de la dépendance, de «permettre l'automatisation de l’analyse» des données et «d'introduire l'automatisme, utilisé depuis longtemps dans l'exploitation descriptive des sondages et enquêtes, au niveau de l'exploitation explicative » (Boudon, I967a, p. I30, I59, 412). Certes, l’Américain Nathan Keyfitz estime 
que «Boudon does talk about simulation, but on the whole Coleman seems more computerminded » (Keyfitz, 1968, p. 634); mais l'on doit rappeler que l'usage des ordinateurs est alors beaucoup plus répandu dans les universités nord-américaines (toutes disciplines confondues) que dans les universités françaises.

\subsection{LES APPORTS ÉPISTÉMOLOGIQUES}

L'intérêt de L'Analyse mathématique ne se limite pas à la présentation d'outils techniques. Dans la tradition durkheimienne', il constitue un manifeste proclamant que la sociologie peut être une science à part entière, à condition de lui conférer la même rigueur que celle des sciences plus anciennes. «L'analyse mathématique des faits sociaux avait en effet pour but [...] d'identifier les fonctions des mathématiques dans les sciences sociales. L'une des plus importantes est celle de la clarification des concepts » (Boudon, 2003, p. 6I).

En outre, son auteur a voulu émanciper la sociologie des conceptions scientifiques héritées des psychologues. On a vu le rôle important que ces derniers ont joué dans la formation technique des sociologues. Mais, alors que la psychologie différentielle se préoccupe essentiellement de décrire et prévoir, la sociologie ambitionne d'expliquer ${ }^{\circ}$. En outre, le psychologue, comme l'agronome, peut expérimenter, en manipulant les variables qu'il suppose influer sur les phénomènes qu'il étudie; dans le cadre d'un plan d'expérience, les structures auxquelles il se réfère, et qu'il a lui-même élaborées, sont donc des structures simples. À l'inverse, le sociologue part en général de faits d’observation sur lesquels, le plus souvent, il ne peut avoir aucune action. Il lui faut traduire ses hypothèses sous la forme d'un modèle explicatif, qu'il doit ensuite confronter à ces données. Compte tenu de la complexité des phénomènes sociaux, ce modèle explicatif ne peut se réduire à une structure simple. Bien que seuls les deux premiers cinquièmes de L'Analyse mathématique soient explicitement consacrés à l’analyse causale, c'est de fait la question de la causalité qui fait l'objet de la quasi-totalité du livre"1.

9 Ou plus précisément dans la tradition de ce que Boudon appelle le «programme TWD (Tocqueville, Weber, Durkheim)», dont il se réclame explicitement (Boudon, 200 lb, p. 373-375).

10 De même, l'économie peut se limiter à prévoir sans nécessairement expliquer.

II La courte troisième partie (p.205-252), consacrée à l'analyse dimensionnelle et à la 


\subsection{LE RÔLE DE LA THÈSE D'ÉTAT EN LETTRES ET SCIENCES HUMAINES}

L'Analyse mathématique marque une étape importante dans la carrière de Boudon. Élu en 1965 maître de conférences à Bordeaux ${ }^{12}$, où il enseigne la sociologie de l'éducation, il souhaite mener de front la recherche et la diffusion des connaissances, et opte pour une carrière universitaire plutôt que de chercheur au CNRS ; mais il lui faut, pour devenir professeur d'université, passer avec succès son Doctorat d’État.

En Lettres et en Sciences, il n'existe pas d'agrégation de l'enseignement supérieur et le recrutement au niveau professoral se fait principalement sur les travaux antérieurs du candidat (enseignement, publications, et thèses de doctorat), qui jouent par conséquent un rôle déterminant dans cette accréditation. En 1967, la thèse de Doctorat d'État n'est plus «le premier travail scientifique important d'un jeune professeur» (Circulaire du I4 novembre 1903). Devenue un exercice obligé pour tout candidat à un poste de professeur d'université, elle a, de ce fait, subi une inflation considérable. À cette époque, le candidat doit encore présenter deux thèses: l'une, dite principale, est censée être un travail original et novateur; l'autre, dite complémentaire, peut être un travail d'érudition, la présentation et la traduction d'un texte inédit, etc. Le volume et la qualité des thèses, la notoriété des directeurs de thèse et des membres du jury, les jugements portés par ceux-ci (consignés dans le rapport de soutenance, alors non communicable à l'intéressé), conditionnent de fait toute la carrière universitaire ultérieure ${ }^{\mathrm{i}}$. De ce point de vue, la thèse principale de Boudon représente un cas exemplaire de l'application de ces critères implicites. Le sujet est

construction des typologies, constitue une rupture dans l'exposé, et n'aborde pratiquement pas la question de l'analyse causale.

12 Après avoir, de 1964 à 1965, remplacé dans cette même université, François Bourricaud pendant le séjour de celui-ci à Harvard.

13 Dans cette perspective, le lectorat visé prioritairement par le candidat n'est pas la communauté sociologique toute entière, mais les membres du jury. II s'agit avant tout de prouver à ces derniers que le postulant à une carrière d'enseignant-chercheur en sociologie possède bien, au plus haut niveau, toutes les compétences requises. Pour la thèse principale, le rapporteur était naturellement le directeur de thèse, Jean Stoetzel, et les deux assesseurs, Roger Daval et Robert Fortet. 
original et novateur, puisqu'il porte sur la pensée formelle appliquée à l'analyse de données d'observation quantifiées; en outre, le candidat fait montre non seulement de la maîtrise de son sujet (y compris de l'outil mathématique), mais aussi d'une connaissance approfondie des grands pionniers de la sociologie.

L'écriture de L’Analyse mathématique a été achevée le $\mathrm{I}^{\mathrm{er}}$ août 1966 . Mais comme la thèse principale devait être imprimée avant la soutenance, celle-ci n’a pu avoir lieu que le I8 mars 1967, à la Sorbonne. La thèse secondaire, Essai sur la signification de la notion de structure dans les sciences humaines, a été publiée en $1968^{14}$. Le jury était composé de trois spécialistes des sciences sociales (Raymond Aron, Roger Daval, Jean Stoetzel), d'un spécialiste de la philosophie des sciences (Georges Canguilhem), et d'un spécialiste du calcul des probabilités (Robert Fortet). À la rentrée de 1967, Boudon était nommé professeur à la Sorbonne sur la chaire de méthodologie des sciences sociales ; or, la nomination d'un Professeur de sociologie de 33 ans à la Sorbonne est un cas tout à fait exceptionnel (Morin, 2006, p. 30). La même année, Boudon succède à Stoetzel à la direction du CES.

\section{L'ACCUEIL RÉSERVÉ PAR LES SOCIOLOGUES À L'ANALYSE MATHÉMATIQUE LORS DE SA PARUTION}

Au cours des mois qui ont suivi la publication de L'Analyse mathématique, on relève deux types de comptes rendus : de courtes notices, résumant le contenu de l'ouvrage et portant un jugement sur celui-ci, et des articles plus détaillés, développant un point particulier.

\section{I. LES SIMPLES COMPTES RENDUS BIBLIOGRAPHIQUES}

Les comptes rendus courts portent tous sur l'ouvrage un jugement globalement positif. Par exemple, Joseph Lajugie conclut sa présentation en ces termes : 
La querelle de l'utilité de la formalisation mathématique en sociologie s'est aujourd'hui renouvelée, car il ne s'agit plus guère d'affirmer ou de nier cette utilité, mais de constater que de nombreux modèles sont restés inappliqués, ou n'ont pas fait l'objet de recherches plus poussées. Il faut aujourd'hui simplement se dire qu'il s'agit là d'un «état de choses inévitable dans une discipline jeune». Le grand mérite quaura eu Raymond Boudon dans cet ouvrage est d'avoir eu conscience de cet état de choses, sans se décourager pour autant et d’avoir persévéré dans cette voie (Lajugie, 1968, p. 587).

Après avoir déploré la rareté, en méthodologie, des ouvrages fondamentaux à visée synthétique, René Bassoul n’hésite pas à affirmer :

Ce livre est appelé à prendre place parmi les meilleurs d'entre eux. Le propos de l'auteur est de traiter des apports de la pensée formelle à la sociologie d'observation et de réhabiliter l'explication causaliste si fréquemment décriée. L'analyse des causes, des dimensions et des processus sont l'objet de ses préoccupations majeures [...]. Cet ouvrage vaut non seulement par l'examen critique et la remise en ordre de méthodes connues mais aussi par l'apport novateur et créateur qu'il comporte, notamment en ce qui concerne l'analyse de la dépendance. Riche en exemples, il sera indispensable aux sociologues et aux méthodologues qui en feront un des tout premiers ouvrages de référence et de travail en méthodologie. Il vaut également par les problèmes qu'il pose et par ceux que se pose l'auteur (Bassoul, 1967, p. 367-368).

Pierre Arnaud constate qu'«il est peu de problèmes de la méthodologie sociologique qui ne s'y trouvent abordés», et souligne la «largeur d'esprit » et la «vaste culture anthropologique, pour ne pas dire philosophique» de l'auteur, «sans lesquelles il ne saurait y avoir de sociologue digne de ce nom».

Décevant pour les seuls esprits étroits et dogmatiques qui confondent les mots et les choses, l'essai de Raymond Boudon est en effet étrangement satisfaisant, et réconfortant, pour ceux qui n'ont jamais cru à aucune harmonie préétablie entre les phénomènes sociaux et tel ou tel langage formel indifférent au contenu qu'on veut lui faire symboliser, et qui ne se fient, au moment de comprendre l'existence sociale, à aucun automatisme explicatif (Arnaud, 1968, p. 186). 
Pour Nathan Keyfitz, ce livre est «a fine account, enthusiastic but not uncritical, of mathematical sociology». Il estime qu'avec les ouvrages de Coleman et de Boudon, les frontières de la sociologie mathématique deviennent plus nettes. De plus, selon lui, ces deux auteurs présentent pour le lecteur l'avantage de limiter leurs propos aux mathématiques élémentaires (Keyfitz, 1968).

Ce dernier point n’est pas l'avis de René Boirel, pour qui il s'agit d'un «ouvrage difficile, dont la lecture exige des connaissances mathématiques étendues». Mais il reconnaît que «le formalisme mathématique rend le grand service d'obliger à une formulation claire de propositions imprécises, et il prend le relai de l'intuition lorsque celle-ci est incapable d'apercevoir toutes les conséquences d'un ensemble de propositions » (Boirel, 1968).

De même, Alberto Comerio di Valenza (1967) ne partage pas l'idée de l'auteur qu'il suffit au lecteur de posséder les connaissances mathématiques et statistiques élémentaires requises des étudiants en sciences sociales; il estime au contraire que la lecture de ce livre, qu'il considère d'ailleurs plus comme un chapitre de la méthodologie que comme un véritable traité, requiert une solide formation en mathématiques.

«Voici un ouvrage dont on peut parier qu'il y sera fait référence dans un proche avenir comme “le Boudon”. L’intérêt premier de ce livre réside en effet dans sa visée synthétique», estime de son côté Jean-Gustave Padioleau, qui salue en ces termes «le premier exposé systématique des méthodes formelles utilisée en sociologie d’observation ». Mais ce compte rendu n'est pas exempt de critiques :

Il est regrettable, néanmoins, que l'auteur de ce bel ouvrage passe sous silence un certain nombre de problèmes que rencontrent les utilisateurs de méthodes formelles (citons, pour simple exemple, la question du degré d’ajustement entre les valeurs observées et celles déduites d'un modèle) ; qu'il laisse dans l'ombre la théorie des jeux dont la valeur heuristique dans l'analyse des phénomènes sociaux ne prête plus guère à contestation et dont les applications à l'analyse sociologique inductive se multiplient (théorie des coalitions). Il va sans dire que la lecture d'un tel ouvrage requiert une solide formation mathématique et statistique; nous sommes convaincus, cependant, que nombre de démonstrations auraient pu être effectuées avec une plus grande simplicité, fût-ce aux dépens de l'élégance mathématique (Padioleau, 1969, p. 948). 


\subsection{LES CRITIQUES CONSTRUCTIVES}

Quelques lecteurs ne se sont pas contentés d'un simple jugement, certes toujours globalement positif, sur l'ouvrage. Ils ont cru nécessaire d’aborder des problèmes techniques difficiles, dont ils pensent qu'ils méritent des ajustements, des compléments, voire des corrections. Nous en citons deux.

Après avoir salué «un ouvrage très important car il apporte aux sociologues une grande richesse de méthodes, en général plus puissantes ou plus fines que celles qui sont couramment mises en pratique», Alain Degenne soulève le problème de l'identification et de l'interprétation des coefficients de régression lorsque la liaison étudiée est de nature statistique (problème abordé dans Boudon, I967a, p. 88 et suiv.). Il souligne que l'utilisation systématique de la méthode d’ajustement par les moindres carrés pour l'estimation des coefficients de régression ne permet pas, in fine, de reconstruire le modèle initial.

Le fait que précisément la méthode ne soit pas réversible, oblige pratiquement à tester tous les modèles compatibles avec la théorie étudiée et ne garantit en rien que le choix effectué soit bon. Il ne ságit d'ailleurs pas d'une caractéristique propre à cette méthode. C'est le cas général pour les analyses de type statistique. Il est important que de nombreuses analyses de dépendance soient faites et que les interprétations puissent être comparées ce qui conduira à une meilleure connaissance de cette méthode qui devrait se révéler très féconde (Degenne, 1967, p. 388).

La contribution de François-André Isambert reprend une discussion déjà entamée avec Boudon à propos des méthodes d’analyse causale :

Rendre à l'explication et à la causalité en sociologie leur dignité scientifique devant un certain défaitisme intellectuel excluant toute interprétation autre que compréhensive ou fonctionnelle, offre un point de départ sain. C'est le signe qu'on ne cherche pas à transiger avec la rigueur sous prétexte de spécificité de l'objet. C'est aussi se contraindre à ne considérer que des relations causales exprimables en langage mathématique: contrainte féconde dans la mesure où elle oblige à la précision dans l'écriture et à la rigueur dans le raisonnement (Isambert, 1967, p. 369-370). 
Mais la traduction en formulations mathématiques des propriétés formelles de la causalité soulève quatre types de difficultés: I) on ne peut réduire une relation causale à une implication logique (une cause peut apparaître comme une implication nécessaire, suffisante, ou les deux) ; 2) toute analyse causale repose sur la pluralité des causes (la cause unique est un cas exceptionnel) ; 3) deux causes ou deux effets ayant même mesure ne sont pas nécessairement équivalents ; 4) hormis dans les cas d'expérimentation, il faut pouvoir mettre en évidence l'indépendance des variations des causes entre elles. «Nous voyons donc qu’à chaque pas se pose le problème des hypothèses statistiques complémentaires des relations fonctionnelles entre les variables, et sans lesquelles une analyse causale partant d'observations simultanées ne peut avoir de sens $»^{15}$. L'hypothèse de la «spécificité des erreurs » étendue à la spécificité des variables implicites (et même des variables exogènes) est, selon Isambert, un postulat apparemment indispensable à toute analyse causale.

La possibilité, qui reste toujours, de poser l'hypothèse minimale, amène à poser l'hypothèse maximale en connaissance de cause, s'il y a lieu, c'est-à-dire lorsqu'elle est matériellement vraisemblable [...]. La vérification porte sur un corps d'hypothèses vraisemblables, les hypothèses d'indépendance statistique de l'un et l'autre type faisant partie de ce corps d'hypothèses au même titre que les hypothèses structurelles (ibid., p. 384).

\subsection{LA RÉPONSE DE BOUDON}

Dans le même numéro de la Revue française de sociologie, Boudon répond en ces termes aux remarques de Degenne et d'Isambert :

Sur le plan de la méthodologie, [...] si les méthodes proposées permettent d'entrevoir le jour où l'automatisme pourra se substituer aux procédures intuitives actuellement utilisées dans l'exploitation des enquêtes sociologiques, un certain nombre de problèmes mathématiques doivent être préalablement résolus (1967b., p. 402).

I5 Ces hypothèses complémentaires sont essentiellement l'hypothèse de clôture, selon laquelle «les facteurs dont les actions ne sont pas contrôlées n'affectent pas simultanément les variables explicatives et les variables à expliquer»; et l'hypothèse de spécificité des facteurs, qui suppose que «les facteurs implicites n'agissent pas simultanément sur deux variables explicatives» (Boudon, 1967a, p. 82). Boudon préfère le terme de «facteurs implicites» à celui d'«erreurs» utilisé par Herbert A. Simon. 
Il n'en demeure pas moins que l'analyse dépend toujours de propositions invérifiables et qu'on ne peut jamais administrer la preuve absolue d'une influence causale dans les situations d'observation caractéristiques de la sociologie. Car les variables impliquées dans l'analyse d'un phénomène social sont toujours à la fois très nombreuses et physiquement indissociables ». Dans les situations nonexpérimentales qui caractérisent la sociologie, il est impossible de démontrer la présence d'une cause; plus précisément, la démonstration dépend toujours de propositions indémontrables (ibid., p. 393).

Les méthodes mathématiques ne servent en aucune manière à combler la distance infranchissable entre les situations expérimentales et les situations non expérimentales. Elles permettent seulement d'analyser des données dont la complexité est telle qu'elles dépassent les ressources de l'intuition (ibid., p. 395).

\section{L'ANALYSE CAUSALE}

Avec le recul, on constate que, pour la plupart des sociologues, l'apport essentiel de L’Analyse mathématique a été la présentation de l'analyse causale. Cette méthode a fait l'objet de nouvelles discussions techniques, et a bénéficié de sérieux efforts de vulgarisation.

\section{I. LES DISCUSSIONS TECHNIQUES}

Comparant les coefficients de régression et les coefficients de dépendance proposés par Boudon, André Régnier met l’accent sur les hypothèses qui sous-tendent la validité des coefficients de dépendance (en particulier, que le graphe correspondant aux relations de causalité soit transitif et sans cycle) et conclut que «les coefficients de régression constituent une solution possible au problème de l’analyse causale, et qu’on peut les nommer dans ce cas coefficients de dépendance» (Régnier, I969, p. Io).

Après avoir rappelé l'importance, dans une structure causale, de l'ordre de présentation des variables ${ }^{16}$, Anselme Flavigny avait souligné les difficultés suscitées par la prise en compte des effets d'interaction dans l'analyse de dépendance (Flavigny, 1969).

16 De l'antériorité des variables explicatives sur les variables expliquées, et de l'ordre chronologique des variables explicatives entre elles. 
Une discussion de Boudon avec Michel Loriaux sur le traitement des effets d'interaction dans la mesure des coefficients de dépendance avait conduit Boudon à corriger, dans la seconde édition de L’Analyse mathématique, son premier exemple de structure causale avec effets d'interaction (Boudon, 1970, p. I23-I27). Mais cette correction n’avait pas convaincu Loriaux, et, après un second échange de points de vue (Loriaux, 1972a; Boudon 1972), il continue à penser que «la méthode défendue par R. Boudon aboutit [...] à la non-interprétabilité des coefficients, liée à la présence d'une variable d'interaction arbitraire que ni les exigences théoriques, ni celles de la statistique ne justifient» (Loriaux, I972b, p. 568) ${ }^{17}$.

\subsection{LES EFFORTS DE VULGARISATION}

Une «présentation élémentaire de cette méthode » à l’usage des sociologues a été proposée dès 1969 par Jacqueline Feldman-Högaasen. Mais c'est surtout Boudon lui-même qui, dans Les Mathématiques en sociologie (Boudon, 1971a) ${ }^{18}$, a développé une initiation à l'analyse causale à la portée d'un bachelier moyen ${ }^{19}$. Après avoir rappelé quelques notions générales de base (effet Condorcet,

17 On notera à ce sujet que Pierre Dubois, qui se réfère explicitement à Boudon dans son application de l'analyse causale à l'explication du taux d'activité des délégués du personnel dans leur entreprise, utilise les coefficients de régression de préférence aux coefficients de dépendance (Dubois, 1970, p. 199-202).

18 Le titre de cet ouvrage ne correspond pas à son contenu réel, mais a été suggéré (imposé?) à l'auteur par les Presses universitaires de France, sans doute parce que jugé plus «vendeur» (communication personnelle de Raymond Boudon à Jean-Paul Grémy, 197I); ce qui a pu parfois entraîner une confusion avec Les Mathématiques. En conséquence, l'auteur a dû préciser dans son Avertissement: «Nous avons choisi dans ce livre d'initiation à la pensée formelle, et à ses utilisations en sociologie, d'approfondir un problème particulier quoique d'importance majeure, plutôt que d'esquisser un rapport général sur les applications des mathématiques à la sociologie. Ce problème est celui de l'analyse des relations d'implication entre les attributs ou plus généralement les variables» (Boudon 197la, p. 5). II insiste à nouveau sur ce point dans son premier chapitre (ibid., p. 18-19), et ne manque pas de rappeler dans sa conclusion sur les limites de son propos: «La matière traitée dans ce volume ne correspond qu'à une infime partie des sujets qu'il aurait été possible de retenir pour une initiation des étudiants en sociologie à l'utilisation de la pensée formelle dans cette discipline» (ibid., p. 265). D'ailleurs, le titre adopté par les éditions Penguin pour la traduction anglaise de cet ouvrage est The Logic of Sociological Explanation, titre qui résume mieux son contenu réel.

19 Les connaissances mathématiques nécessaires pour suivre les démonstrations sont celles qui étaient au programme de la première partie du baccalauréat général de l'époque. 
axiomatique, causalité, implication faible, corrélation), il initie progressivement le lecteur à des schémas de causalité de plus en plus complexes (jusqu’à cinq variables, avec éventuellement des interactions). Mais tous les exemples auxquels il se réfère ne portent que sur des variables dichotomiques (attributs), ce qui en rend la compréhension plus aisée.

En revanche, on peut s'étonner du peu de place qu'il accorde à l'analyse causale dans son «Que sais-je?» sur les méthodes en sociologie: seules quelques pages y sont consacrées aux relations entre variables (Boudon, 1969, p. 58-7I) et à l'analyse multivariée (ibid. p. 79-85), et le terme d'analyse causale n’apparaît pas. Il fait par contre le titre du premier chapitre de sa réédition avec Renaud Fillieule, mais, dans ce chapitre, les passages consacrés aux relations entre variables et à l'analyse multivariée ont été repris de la première édition avec seulement quelques modifications mineures (Boudon et al., 20I2, p. 27-80). En outre, les autres notions abordées dans ces Que sais-je ${ }^{20}$ n'avaient pas été traitées dans L’Analyse mathématique.

\section{L'IMPORTANCE DE L'ANALYSE MATHÉMATIQUE}

Cinquante années après sa publication, quel regard peut-on porter sur L’Analyse mathématique des faits sociaux?

\section{I. UN LIVRE D'ACCÈS DIFFICILE}

Pour la majorité des sociologues français, compte tenu du faible niveau en mathématiques et en statistiques de la majorité d'entre eux, L'Analyse mathématique n’a certainement pas été un livre d’accès facile ${ }^{21}$; et ce, même si certains passages techniques avaient auparavant été développés sous une forme plus accessible

20 La préparation d'une enquête quantitative et les méthodes qualitatives pour le premier, l'individualisme méthodologique et la rationalité cognitive pour le second.

21 Boudon estime pourtant que «la lecture de ce livre, même si elle n'est pas aisée, devrait donc être accessible à toute personne possédant la formation mathématique et statistique élémentaire aujourd'hui requise des étudiants en sciences sociales» (Boudon, 1967a, p. 18). Dans les bibliographies de ses publications ultérieures, il le présente comme une «vue générale sur les méthodes mathématiques de la sociologie quantitative» (Boudon, 197la, p. 267), et considère que sa lecture suppose seulement que l'on connaisse déjà les méthodes élémentaires utilisées dans l'analyse des enquêtes sociologiques (Boudon, 1969, p. 127). À noter 
dans des publications antérieures auxquelles le texte renvoie occasionnellement ${ }^{22}$. D’autre part, la réédition corrigée de 1970 n’a pas eu pour objectif d'en augmenter la lisibilité. Les principales modifications portent sur les formules mathématiques de l'analyse quantitative des variables discontinues (Boudon, 1970, p. 54, 55, 58, 66, 67, 7I), de l’analyse de dépendance (ibid., p. 86, 97, I23-I27, I33), et l'analyse des structures causales simples (ibid., p. I44) ${ }^{23}$.

Ces difficultés d'accès pour les non mathématiciens ont fortement limité son impact auprès des sociologues français, comme le souligne Jean-Michel Morin : il n'y a guère que la communauté scientifique internationale chez qui cet ouvrage a suscité «des discussions sur le passage d'une analyse causale à une approche plus souple en termes de dépendance» (2006, p. 276) ${ }^{24}$.

\subsection{LE JUGEMENT RÉTROSPECTIF DE BOUDON SUR SA THĖSE PRINCIPALE}

Boudon lui-même a contribué à minimiser l'importance de cette première étape dans sa démarche scientifique, en déclarant à Yao Assogba :

[C'est] une thèse qui n'est pas originale en soi, mais qui représente l'effort que j’ai voulu faire pour dresser une sorte de bilan de l'utilité des mathématiques pour les sciences sociales en général et la sociologie en particulier. Mais à la fin de ma thèse, je dois avouer que mon bilan m’a un peu désillusionné [...]. Il ne faut pas

toutefois que, dans la mise à jour de son Que sais-je? sur les méthodes en sociologie, L'Analyse mathématique a été retirée de la bibliographie (Boudon et al., 2012, p. 125) et n'est plus mentionnée que dans une note de bas de page relative à l'analyse de dépendance (ibid., p. 40).

22 En particulier dans les deux anthologies publiées avec Lazarsfeld (Boudon et al., 1966 et 1968), et dans divers articles, comme celui sur les abandons de poursuite judiciaire (Davidovitch et al., 1964).

23 En revanche, les erreurs de classement dans l'Index des noms (ibid., p. 450-45I) n'ont pas été corrigées.

24 Dans son tableau sur l'impact des publications de Boudon, Morin montre que seule la communauté scientifique internationale a réagi lors de la parution de L'Analyse mathématique; ni les étudiants et enseignants français, ni les intellectuels et journalistes parisiens, n'ont manifesté d'intérêt pour les thèmes abordés dans cet ouvrage (Morin, 2006, p. 274). D'ailleurs, dans les références que Morin énumère à ce propos, aucune n'a pour auteur un chercheur français (ibid., p. 276). Pourtant, Michel Loriaux estime que la réédition rapide de cet ouvrage atteste de son succès auprès des chercheurs et des étudiants en sciences sociales (Loriaux, 1972a, p. 550). En 197I, Boudon (197Ib, p. 312) annonce une traduction anglaise de L'Analyse mathématique qui ne verra pas le jour. 
attendre des mathématiques qu'elles soient l'instrument qui puisse permettre à la sociologie de se solidifier. C'est donc à partir de ce bilan que j’ai abandonné un peu la piste de l'analyse mathématique des faits sociaux (Assogba, 1999, p. I34).

Il ajoute: «Je suis sorti du bilan que j’avais dressé dans ma thèse avec l'impression que les mathématiques ne joueraient jamais en sociologie qu’un rôle relativement modeste» (Boudon, 2003, p.4I). «Les rapports entre mathématiques et sciences sociales ayant beaucoup évolué depuis, mon livre est aujourd'hui périmé. Il garde toutefois l'intérêt de porter témoignage sur la conjoncture intellectuelle caractérisant les sciences sociales de l'époque » (ibid., p. 40).

De plus, alors que, exception faite de la courte troisième partie, L'Analyse mathématique traite essentiellement de l'analyse causale, il estime finalement que le potentiel explicatif de l'analyse causale est limité, et que «l'individualisme méthodologique permet de formuler de véritables explications des phénomènes, alors que l'analyse causale s'en tient aux descriptions » (Boudon et al., 20I2, p. 4I-42).

\section{CONCLUSION}

Cet ouvrage peut être considéré comme constituant les fondations des travaux ultérieurs de Boudon. Mais, comme en architecture, les fondations suscitent moins d'intérêt que les superstructures, bien que ce soient elles qui assurent la solidité de l'édifice. À l'époque où ce livre a été publié, il a contribué à dégager la sociologie de l'emprise de la philosophie, en prônant l'application de méthodes scientifiques qui avaient fait leurs preuves dans les disciplines plus anciennes. L’affirmation que l'objectif de la sociologie doit être d'élaborer des modèles explicatifs, destinés à être confrontés à des données d'observation, ouvre une voie médiane entre d'une part les théories purement spéculatives, et d'autre part les essais littéraires, journalistiques, voire militants («Les sciences sociales sont des sciences comme les autres, chargées de produire du savoir sur le social et non d'influencer l'opinion », propos rapportés par Vautier, 2002, p. I3). 
On ne peut que regretter que, par la trop grande technicité requise pour une thèse de Doctorat d'État, ce texte fondamental ait pu sembler inaccessible à des sociologues ne disposant pas de connaissances mathématiques suffisantes. Pourtant, il ne constitue pas seulement, comme le dit Boudon, un «témoignage sur la conjoncture intellectuelle de l'époque»; il devrait être considéré plutôt comme une réaction contre cette conjoncture, une sorte de Discours de la méthode, revendiquant pour la sociologie un statut scientifique égal à celui des autres sciences d’observation. En outre, en présentant, dans son dernier chapitre ${ }^{25}$, le modèle de simulation du mécanisme des abandons de poursuite judiciaire (Davidovitch et Boudon, I964), Boudon a, sans en avoir pleinement conscience à l'époque, posé les premières bases de ce qui deviendra l’individualisme méthodologique (Boudon, 20ı, p. ı8-ı9).

\section{BIBLIOGRAPHIE}

ARNAUD P., 1968, «L'Analyse mathématique des faits sociaux», L'Année sociologique, 19, p. $186-187$.

ASSOGBA Y., 1999, La Sociologie de Raymond Boudon, Montréal, Presses de l'université Laval.

BARBUT M., 1967, Mathématiques des sciences humaines, Paris, PUF.

BASSOUL R., 1967, «L'analyse mathématique des faits sociaux de Raymond Boudon», Revue française de sociologie, 8-3, p. 367-368.

BOIREL R., 1968, «Raymond Boudon, L'Analyse mathématique des faits sociaux», Les Études philosophiques, I, p. 63.

BOUDON R., 1967a, L'Analyse mathématique des faits sociaux, Paris, Plon.

-, 1967b, «Les relations causales: problèmes de définition et de vérification», Revue française de sociologie, 8-3, p. 389-402.

-, 1969, Les Méthodes en sociologie, Paris, PUF.

-, 1970, L’Analyse mathématique des faits sociaux, 2édition revue et corrigée, Paris, Plon. 
-, 197la, Les Mathématiques en sociologie, avec la collaboration de J.-P. Grémy, Paris, PUF.

-, 197Ib, La Crise de la sociologie, Genève, Droz.

-, 197Ic, «Data-Processing and the Use of the Results of Sociological Surveys», International Social Sciences Journal, 23-2, 163-174.

-, 1972, «Un concept bien difficile: I'interaction», Revue française de sociologie, 13-4, p. 564-565.

-, 200 la, «Pourquoi devenir sociologue? Réflexions et évocations», Revue européenne des sciences sociales, 39-120, p. 5-30.

-, 200 lb, «Sociology that Really Matters», European Sociological Review, 18-3, p. 37I-378.

-, 2003, Y a-t il encore une sociologie? Entretiens avec Robert Leroux, Paris, Odile Jacob.

-, 20I0, La Sociologie comme science, Paris, La Découverte.

BOUDON R. et FILLIEULE R., 2012, Les Méthodes en sociologie, Paris, PUF.

BOUDON R. et LAZARSFELD P.F., 1966, L'Analyse empirique de la causalité, Paris, Mouton.

-, 1967, Le Vocabulaire des sciences sociales. Concepts et indices, Paris, Mouton.

CHENU A., 2002, «Une institution sans intention. La sociologie en France depuis l'après-guerre», Actes de la recherche en sciences sociales, I4I-142, p. 46-59.

COLEMAN J. S., 1964, Introduction to Mathematical Sociology, New York, The Free Press of Glencoe.

COMERIO DI VALENZA A., 1967, «Boudon R., L'Analyse mathématique des faits sociaux 》, Studi di Sociologia, 5-4, p. 392-393.

DAVAL R. et GUILBAUD G-Th., 1950, La Méthode statistique. Première partie: statistique à un seul caractère, Paris, PUF.

DAVIDOVITCH A. et BOUDON R., 1964, «Les mécanismes sociaux des abandons de poursuite: analyse expérimentale par simulation», L’Année sociologique, p. III-244.

DEGENNE A., 1967, «Problèmes d'identification et d'interprétation», Revue française de sociologie, 8-3, p.385-388. 
DROUARD A. 1982, «Réflexions sur une chronologie: Le développement des sciences sociales en France de 1945 à la fin des années soixante», Revue française de sociologie, 23-I, p.55-85.

DUBOIS P., 1970, «Un exemple d'analyse causale: l'activité du délégué du personnel dans l'entreprise», Revue française de sociologie, II-2, p. 197-210.

EHRLICH S. et FLAMENT Cl., 196I, Précis de statistique, Paris, PUF.

FAVERGE J.-M., 1950, Méthodes statistiques en psychologie appliquée, Paris, PUF.

FELDMAN-HÖGAASEN J., 1969, «Analyse de dépendance, régression et corrélation», Revue française de sociologie, 10-3, p. 318-332.

FLAMENT Cl., 1965, Théorie des graphes et structures sociales, Paris, Mouton, Gauthier-Villars.

FLAVIGNY A., 1969, «Notes sur l'analyse de dépendance», Revue française de sociologie, 10-3, p. 352-362.

GINER S., 2013, «Raymond Boudon (1934-2013)», Revista Internacional de Sociologia, p. 697-699.

GURVITCH G., 1950, La Vocation actuelle de la sociologie: vers une sociologie différentielle, Paris, PUF.

-, 1957, La Vocation actuelle de la sociologie, nouvelle édition, Tome I, Vers une sociologie différentielle, Paris, PUF.

- (dir.), 1958, Traité de sociologie, Tome I, Paris, PUF.

HEILBRON J., 1991, «Pionniers par défaut? Les débuts de la recherche au Centre d'études sociologiques (1946-1960)», Revue française de sociologie, 32-3, p. 365-379.

HÉRAN F., 2013, «In memoriam Raymond Boudon (1934-2013)», Revue européenne des sciences sociales, 5I-2, p.7-II.

ISAMBERT F.-A., 1967, «Traduction automatique et vérification de quelques systèmes de relations causales», Revue française de sociologie, 8-3, p. 369-384.

KEMENY J. G., SNELL J. L. et THOMSON G. L, 1960, Algèbre moderne et activités humaines, Paris, Dunod.

KEYFITZ N., 1968, «L'Analyse mathématique des faits sociaux by Raymond Boudon», The American Journal of Sociology, 73-5, p.633-634. 
LAJUGIE J., 1968, «L'Analyse mathématique des faits sociaux», Revue d'histoire économique et sociale, 46-4, p. 586-587.

LAUDE Cl., 1960, «Le Centre d'études sociologiques», Revue française de sociologie, I-I, p. 93-97.

LORIAUX M., 1972a, «L'analyse causale face aux effets d'interaction; réexamen de la méthode de dépendance de Raymond Boudon», Revue française de sociologie, 13-4, p.550-563.

-, 1972b, «Réponse à R. Boudon», Revue française de sociologie, 13-4, p.566-568.

MATALON B., 1965, L'Analyse hiérarchique, Paris, Mouton, Gauthier-Villars.

MARCEL J.-Ch., 2005, «Le déploiement de la recherche au Centre d'études sociologiques», La Revue pour l'histoire du CNRS, 13.

MARTIN O., 2002, «Mathématiques et sciences sociales au XXe siècle», Revue d'histoire des sciences humaines, 6, p. 3-13.

MESURE S., 2013, «Hommage à Raymond Boudon», Sociologie, 3-4, p. 247-249.

MORICE E. et CHARTIER F., 1954, Méthode statistique, Paris, Imprimerie nationale.

MORIN J.-M., 2006, Boudon. Un sociologue classique, Paris, L'Harmattan.

PADIOLEAU J.-G., 1969, «L'Analyse mathématique des faits sociaux», Annales, 24-4, p. 947-948.

PROST A., 1989, « 1968: Mort et naissance de l'université française», Vingtième siècle. Revue d'histoire, 23, p.59-70.

RÉGNIER A., 1969, «À propos de l'analyse de dépendance», Mathématiques et Sciences humaines, 7-25, p.5-1I.

REUCHLIN M., 1962, Les Méthodes quantitatives en psychologie, Paris, PUF.

TRÉANTON J.-R., 1991, «Les premières années du Centre d'études sociologiques (1946-1955)», Revue française de sociologie, 32-3, p. 381-404.

VALLET L.-A., 2013, «Raymond Boudon, in memoriam "Un sociólogo que creía en el individuo"», Revista Española de Sociología, 20, p.9-12.

VAUTIER Cl., 2002, Raymond Boudon. Vie, Oeuvres, Concepts, Paris, Ellipses. 